\title{
Genetic and specific environmental risk factors affected comorbidity of major depression and alcoholism
}

Prescott CA, Aggen SH, Kendler KS. Sex-specific genetic influences on the comorbidity of alcoholism and major depression in a population-based sample of US twins. Arch Gen Psychiatry 2000 Aug;57:803-11.

\section{QUESTION: How much influence do genetic and environmental risk factors have on comorbidity of major depression and alcohol abuse or dependence?}

Design

A population based, cohort study using data from a twin registry.

\section{Setting}

Virginia, USA.

\section{Participants}

Participants were from 2 twin studies: 1945 women from a female-female pair study and 6847 participants from a male-male and male-female pair study (mean age 35 y). 8733 participants were included in the individual level analyses; twin pair analyses were based on 7460 participants from complete pairs with known zygosity and diagnostic data. Complete pairs included 862 monozygotic male, 506 monozygotic female, 649 dizygotic male, 330 dizygotic female, and 1408 opposite sex pairs.

\section{Assessment of risk factors}

A liability threshold model was used to estimate the genetic and environmental contributions to twin-pair liability to depression and alcoholism. 3 sources were considered: additive genetic (genes whose allelic effects combine additively), common environment (all prenatal and postnatal environments shared by twin pair members), and specific environment (all remaining factors not shared by twin pair members). Sex differences were included in all models. Members of a twin pair were interviewed by interviewers who were blind to co-twin diagnosis.

\section{Main outcome measures}

Lifetime major depression, alcohol abuse, and alcohol dependence were assessed by standard structured interviews for DSM-III-R and DSM-IV diagnoses.

\section{Main results}

Within person correlations of liabilities for depression and alcohol disorders ranged from 0.24 to 0.31 in men and from 0.29 to 0.37 in women. Twin pair similarity for depression and alcohol disorders was strongest among monozygotic pairs compared with same sex and opposite sex dizygotic pairs. Cross twin, cross disorder correlations were higher in monozygotic pairs as opposed to dizygotic pairs, ranging from 0.20 to 0.21 in men and from 0.15 to 0.20 in women. When models for comorbidity were applied, the best fit model was the one positing that there are sex specific genetic and specific environmental factors that contribute jointly to comorbidity of alcoholism and depression, as well as genetic and specific environmental factors that contribute only to alcoholism or only to depression. In this model, genetic factors accounted for $61 \%$ of the association in men and for $51 \%$ in women. The genetic factors that contributed to comorbidity in women were independent, however, of those contributing to comorbidity in men.

\section{Conclusions}

Genetic and specific environmental risk factors contributed to the comorbidity of major depression and alcohol abuse or dependence. Different factors operated in men and women.

\section{COMMENTARY}

This study by Prescott et al broadly supports previous findings, although the detailed methodology is not for the idly curious! The basic findings were that alcohol dependence and depression were linked, more than by chance, both within and between people and their same sex twins, although the overlap was not large. Both genes and environment were important, but genes had slightly more influence on comorbidity. Of clinical relevance was that depression in comorbid men and women had an earlier age of onset than "pure" depression, suggesting that comorbid depression was more severe. Both conditions were common in this sample: fully half of the participants had depression, alcohol abuse or dependence, or both. This, more than ever, emphasises how much of the treatment of both of these conditions falls within the realm of primary care.

Striking sex differences existed: men drank more and women were more depressed. Comorbidity was more common in men, with comorbid depression and alcohol abuse or dependence being as common as either "pure" disorder, whereas pure depression was more common in women However, of those (relatively few) women with alcohol dependence or abuse, nearly three quarters had depression as well. A difference also existed in the order in which the conditions began: women reported depression onset before drinking, while men showed drinking problems earlier than depressive problems (to a non-significant extent), but on self report were more likely to state that the drinking came first. Unfortunately, order of onset was not used to decide which disorder was "secondary." The authors instead defined secondary alcoholism as occurring only within episodes of depression, which will weaken the links, because once established, alcohol dependence functions as a separate disorder and occurs spontaneously irrespective of its initial causation. This also excludes the possibility of "secondary depression," which is at least a possibility in men. Nevertheless, it seems that in men, alcohol dependence tends to be a precursor to depression, whereas in women it is a consequence. These sex differences were further highlighted in the factor analysis, which strongly suggests that comorbidity of alcohol dependence and depression have different genetic causes in men and women.

Pete Sudbury, BM, BCh, MRCPsych, MBA Heatherwood and Wexham Park Hospitals Trust Slough, Berkshire, UK 